\title{
Influencia del secado en el factor de potencia del aislamiento de los arrollamientos de transformadores de distribución
}

\section{Influence of drying on the insulation power factor of distribution transformer windings}

\author{
Jorge Ramos ${ }^{1 *}$, Carlos García' \\ ${ }^{1}$ Facultad de Ingeniería Eléctrica y Electrónica, Universidad Nacional de Ingeniería. Lima, Perú \\ Recibido (Received): 10/09/2018 Aceptado(Accepted): 11/09/2018
}

\begin{abstract}
RESUMEN
El presente artículo expone el desarrollo de la investigación sobre el análisis y determinación de la influencia del proceso de eliminación de la humedad, absorbida por el aislamiento sólido de los arrollamientos, en el factor de potencia del aislamiento de los transformadores de distribución. Considerándose al factor de potencia como un parámetro característico que permite determinar qué tan seco está el aislamiento y definir así su calidad desde el punto de vista eléctrico. Se demostró que existe una influencia significativa sobre el factor de potencia.
\end{abstract}

Palabras clave - Secado en vacío, factor de potencia del aislamiento, aislamiento de los arrollamientos, capacitancia de los arrollamientos.

\section{ABSTRACT}

The present article exposes the development of the investigation on the analysis and determination of the influence of the process of elimination of the humidity, absorbed by the solid isolation of the windings, in the power factor of the isolation of the distribution transformers. Considering the power factor as a characteristic parameter that allows to determine how dry the insulation is and thus define its quality from the electrical point of view. It was shown that there is a significant influence on the power factor.

Keywords - Vacuum drying, insulation power factor, insulation of windings, capacitance of windings.

\section{INTRODUCCIÓN}

Los transformadores de distribución en baño de aceite dieléctrico son máquinas eléctricas utilizadas para el suministro de energía eléctrica en sistemas de distribución. Constructivamente están conformados por el tanque, la parte activa y los accesorios normales, siendo un componente adicional el aceite dieléctrico (que actúa como refrigerante y aislante).

La parte activa es el componente más importante del transformador y está constituida por el núcleo magnético, los arrollamientos y los elementos de conexionado. Durante el proceso de fabricación de los arrollamientos, y posterior montaje en el núcleo magnético, sus aislamientos absorben humedad del medio ambiente.

\footnotetext{
${ }^{*}$ Correspondencia:
}

E-mail: jorgeramosc@uni.edu.pe

doi: https://doi.org/10.21754/tecnia.v28i2.349
Este exceso de agua en el material aislante, si no se elimina adecuadamente, producirá el envejecimiento acelerado ante la presencia de altas temperaturas de operación, poniendo en riesgo la vida útil e integridad del transformador.

Por esta razón, la parte activa se somete a un proceso de eliminación de la humedad (secado del aislamiento), que generalmente se realiza en un horno con circulación de aire caliente y a presión atmosférica por un tiempo determinado. En este proceso, la medición del factor de potencia del aislamiento sólido de los arrollamientos y su comparación con valores experimentales, y recomendaciones de las normas técnicas, permiten determinar qué tan seco está el aislamiento y definir así su calidad desde el punto de vista eléctrico. 
Un proceso complementario de secado es el tratamiento a presión de vacío del transformador, antes de la impregnación en aceite dieléctrico, con mediciones del factor de potencia del aislamiento sólido de los arrollamientos. En dicho proceso es necesario, a partir de los resultados de las mediciones, analizar y determinar los efectos producidos en la calidad del secado del aislamiento. En este contexto se formulan el problema general ¿Cómo interactúan el aislamiento seco de los arrollamientos y el tiempo del tratamiento en vacío sobre el factor de potencia del aislamiento de los transformadores de distribución? y los problemas específicos: ¿Qué efecto produce el aislamiento seco de los arrollamientos en el factor de potencia del aislamiento de los transformadores de distribución? ¿Qué efecto produce el tiempo del tratamiento en vacío en el factor de potencia del aislamiento de los transformadores de distribución?

Desde el punto de vista aplicativo y académico, el estudio es importante porque analiza el proceso de secado en vacío y su influencia en el factor de potencia del aislamiento de los arrollamientos de transformadores de distribución utilizando la metodología de la investigación científica y la aplicación del análisis estadístico; contribuyendo así con el desarrollo de los conocimientos técnicos con base teórica. Centra el análisis en el proceso de secado de la parte activa de cuatro transformadores trifásicos de distribución, con núcleo magnético tipo columnas y de 500 Kilovoltioamperios (KVA) de potencia, de fabricación nacional.

Se establece como objetivo general, determinar el efecto que produce la interacción del aislamiento seco de los arrollamientos y el tiempo del tratamiento en vacío sobre el factor de potencia del aislamiento de los transformadores de distribución. $Y$ como objetivos específicos, determinar el efecto que produce el aislamiento seco de los arrollamientos en el factor de potencia del aislamiento de los transformadores de distribución, y determinar el efecto que produce el tiempo del tratamiento en vacío en el factor de potencia del aislamiento de los transformadores de distribución.

Para dar respuesta a las preguntas de investigación se formulan la hipótesis general $\boldsymbol{H}_{\mathbf{1}}$ : El aislamiento seco de los arrollamientos y el tiempo del tratamiento en vacío interactúan sobre el factor de potencia del aislamiento de los transformadores de distribución; y las hipótesis específicas $\boldsymbol{H}_{2}$ : El aislamiento seco de los arrollamientos produce efecto significativo sobre el factor de potencia del aislamiento de los transformadores de distribución; y $\boldsymbol{H}_{3}$ : El tiempo del tratamiento en vacío produce efecto significativo sobre el factor de potencia del aislamiento de los transformadores de distribución. Definiéndose como doi: https://doi.org/10.21754/tecnia.v28i2.349 variables independientes el aislamiento seco de los arrollamientos, que son los diferentes aislamientos sólidos de los arrollamientos de la parte activa del transformador de distribución; y el tiempo del tratamiento en vacío, que es la duración en horas del proceso en vació, a un milibar de presión, al que es sometida la parte activa dentro de su tanque. Y como variable dependiente el factor de potencia del aislamiento, que viene a ser el valor que determina el grado de secado del aislamiento sólido de los arrollamientos de la parte activa del transformador de distribución en el proceso de tratamiento en vacío.

\section{BASE TEÓRICA}

\subsection{Factor de Potencia del Aislamiento}

El factor de potencia es un parámetro del material dieléctrico que constituye el sistema de aislamiento de las máquinas eléctricas, y en particular de los transformadores de distribución, el cual representa las pérdidas eléctricas totales en el volumen de este material, y que son el resultado de los diferentes fenómenos que se presentan en la estructura del sistema de aislación. Con el fin de tener una mejor comprensión del concepto de factor de potencia se plantea un modelo del sistema de aislación basado en parámetros eléctricos concentrados. Este modelo representa todos aquellos procesos físicos que determinan el comportamiento eléctrico macroscópico del aislamiento.

Para realizar el modelo se deberá tener en cuenta que al aplicar una excitación de tensión alterna al sistema de aislación, circulará por éste una corriente total que explica los siguientes procesos físicos [1]:

- Capacitancia geométrica del sistema de aislamiento: Los dieléctricos dependiendo de su forma constructiva presentan efectos capacitivos entre sus componentes. En una condición ideal pueden modelarse como un capacitor [1].

- Polarización del dieléctrico: El modelo físico para estos procesos estará constituido por resistores y capacitores. Estos parámetros concentrados involucran los procesos físicos de pérdidas, magnitud de la polarización y tiempo de relajación [1].

- Conducción superficial y volumétrica: Se debe al movimiento continuo de los portadores de carga a través del sistema de aislación. Depende de la temperatura, humedad, contaminación, nivel de la tensión aplicada, calidad y estado del aislamiento. Se modela este comportamiento mediante un resistor [1].

- Ionización: Comprende el proceso físico de las descargas parciales, superficiales o internas, que Revista TECNIA Vol. 28 N 1 Agosto - Diciembre 2018 
consumen energía y que a su vez producen el aumento de la capacitancia del sistema de aislación. El modelo de este proceso estará constituido por un resistor y un capacitor [1].

Por lo expuesto, el sistema de aislamiento de los transformadores puede modelarse como un capacitor con pérdidas, que se representa mediante un circuito eléctrico de parámetros concentrados conformado por un capacitor ideal $\boldsymbol{C}_{\boldsymbol{d}}$ en paralelo con un resistor $\boldsymbol{R}_{\boldsymbol{d}}$, ver Figura 1.
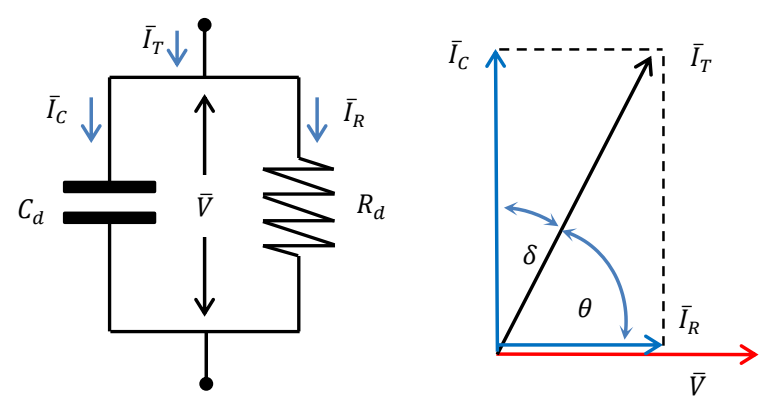

Figura 1. Modelo del aislamiento.

Cuando un voltaje $\overline{\boldsymbol{V}}$, de una fuente de tensión, se aplica a través del aislante, la corriente fasorial $\overline{\boldsymbol{I}}_{T}$ que circula tendrá dos componentes denominados $\overline{\boldsymbol{I}}_{\boldsymbol{C}}$ (a través del capacitor $\boldsymbol{C}_{\boldsymbol{d}}$ ) e $\overline{\boldsymbol{I}}_{\boldsymbol{R}}$ (a través del resistor $\boldsymbol{R}_{\boldsymbol{d}}$ ). Un aislante ideal tendría $\overline{\boldsymbol{I}}_{\boldsymbol{T}}=\overline{\boldsymbol{I}}_{\boldsymbol{C}}$, lo cual significaría que la corriente que entrega la fuente estaría adelantada $90^{\circ}$ a la tensión aplicada. Un buen aislamiento eléctrico tendrá un ángulo de desfasaje muy cercano a $90^{\circ}$. Cualquier deterioro en el aislamiento producirá un aumento de la desviación angular $\boldsymbol{\delta}$, ver Figura 1. Definiéndose entonces el factor de potencia y la tangente delta como:

$$
\begin{gathered}
\cos \theta=\frac{I_{R}}{I_{T}} \\
\operatorname{tg} \delta=\frac{I_{R}}{I_{C}}
\end{gathered}
$$

En general se deberá tener presente que el factor de potencia y la tangente delta tienen valores similares cuando el ángulo $\boldsymbol{\theta}$ es, aproximadamente, mayor a $80^{\circ}$, siendo diferentes cuando es menor a este valor [2].

Según recomendaciones de la norma internacional [3], los transformadores nuevos en baño de aceite dieléctrico deben tener valores de factor de potencia menor al 0,5\%, medidos a una temperatura de $20{ }^{\circ} \mathrm{C}$. Para el aislamiento seco de los arrollamientos, sin impregnación de aceite dieléctrico, las normas no prescriben un valor de referencia, por tanto en base a la experiencia se puede considerar como un valor aceptable el que refiere la norma mencionada [3]. Si la temperatura de medición difiere de $20^{\circ} \mathrm{C}$ es necesario corregir por temperatura los valores medidos del factor doi: https://doi.org/10.21754/tecnia.v28i2.349 de potencia, según prescribe la norma para transformadores inmersos en aceite dieléctrico [4]. En el caso del aislamiento seco de los arrollamientos, sin impregnación en aceite dieléctrico, la norma no prescribe un valor de corrección por temperatura.

En la realización de las mediciones del factor de potencia del aislamiento y de la capacitancia de los arrollamientos se utilizan los siguientes modos de prueba:

a) Modo de Prueba de Espécimen a Tierra (GST): La prueba de espécimen a tierra, se conoce como la medición de una muestra de aislamiento que tiene uno de sus terminales conectado a tierra [2]. Para la medición en transformadores de dos arrollamientos, este modo de prueba se caracteriza por que la forma de conexión entre el instrumento de medición y el transformador bajo ensayo logra medir las corrientes y las pérdidas a tierra de los arrollamientos, por tanto, se miden el factor de potencia y la capacitancia de los arrollamientos de alta tensión a tierra, Figura 2, y de los arrollamientos de baja tensión a tierra, Figura 3.

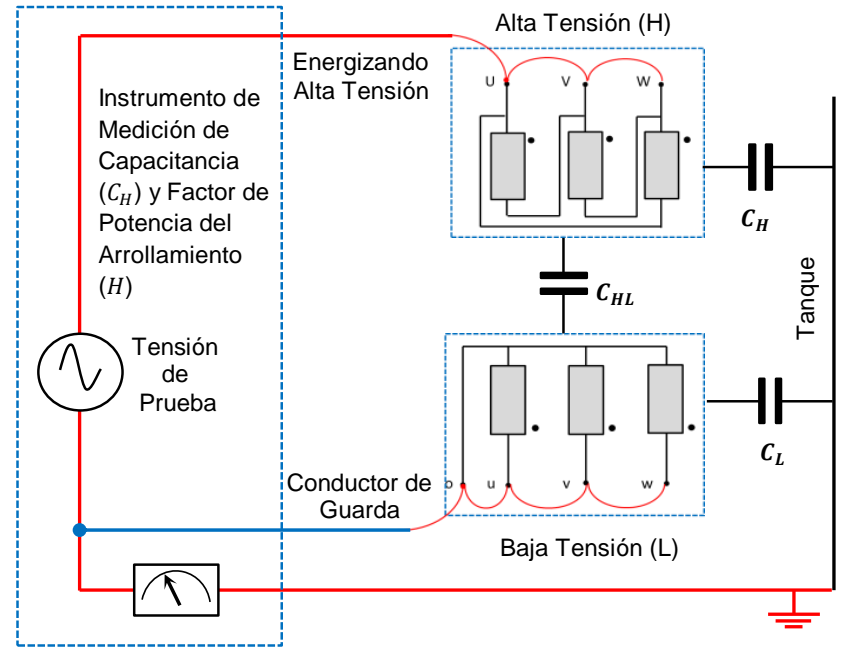

Figura 2. Modo de prueba GST para mediciones en el arrollamiento de alta tensión.

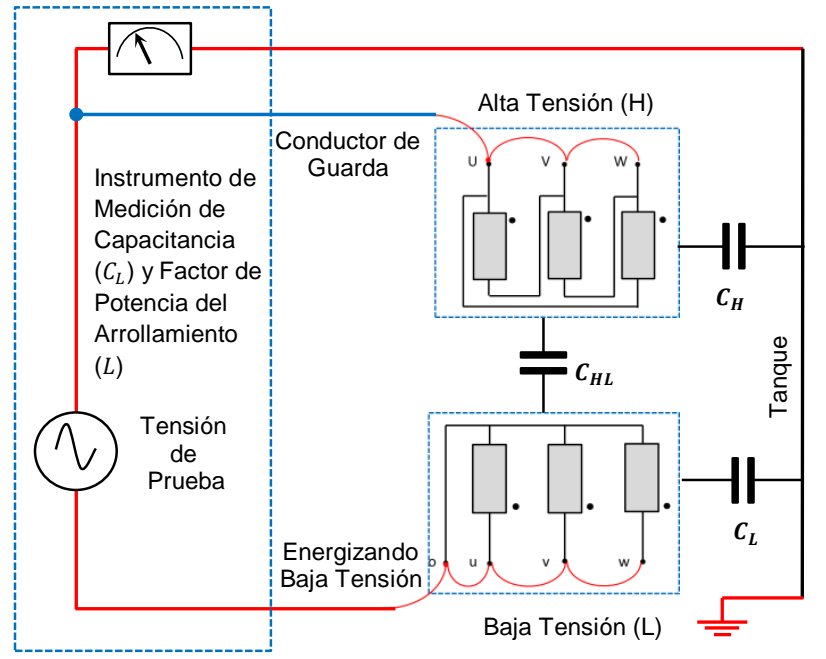

Revista TECNIA Vol. 28 N 1 Agosto - Diciembre 2018 
Figura 3. Modo de prueba GST para mediciones en el arrollamiento de baja tensión.

b) Modo de Prueba de Espécimen no Aterrado (UST): La prueba de espécimen sin conexión a tierra se conoce como la medición de una muestra de aislamiento que no está conectada a tierra [2]. Para la medición en transformadores de dos arrollamientos, este modo de prueba se caracteriza por que la forma de conexión entre el instrumento de medición y el transformador bajo ensayo logra medir las corrientes y las pérdidas entre los arrollamientos, por tanto, se miden el factor de potencia y la capacitancia entre los arrollamientos de alta tensión y los arrollamientos de baja tensión. No mide las corrientes y pérdidas a tierra, ver Figura 4.

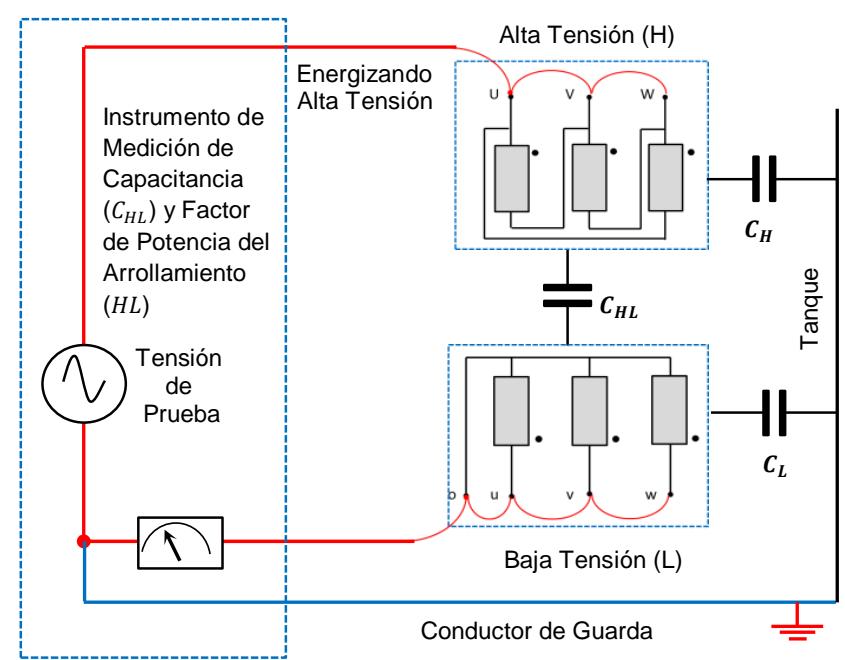

Figura 4. Modo de prueba UST para mediciones entre arrollamientos de alta y baja tensión.

En las figuras, $C_{H}$ y $C_{L}$ son las capacitancias de los arrollamientos de alta tensión a tierra y de baja tensión a tierra, y $C_{H L}$ la capacitancia entre arrollamientos.

\subsection{Eliminación de la Humedad de la Parte Activa}

Los aislamientos sólidos de los arrollamientos de transformadores de distribución, están compuestos principalmente por material celulósico y madera, los cuales llegan a representar el 95\% de los aislamientos. Estos materiales son altamente higroscópicos conteniendo de $8 \%$ a $10 \%$ de su peso en humedad, en malas condiciones de operación. Dentro de ellos el papel aislante seco absorbe agua más rápidamente que el papel impregnado de aceite dieléctrico (el cual tiene un rango más bajo de absorción de humedad). Esta humedad en el papel aislante afecta su rigidez dieléctrica, el factor de potencia y la resistencia mecánica [5], originando el envejecimiento prematuro del material.

doi: https://doi.org/10.21754/tecnia.v28i2.349
Para eliminar la humedad de los aislamientos es necesario transformarla en vapor y expulsarla a la atmosfera; ello se puede lograr aplicando el Método con Presión de Vacío. Este método consiste en un proceso de disminución de la temperatura de ebullición del agua por medio del alto vacío, o sea, bajar la presión interna en el tanque del transformador, que contiene a la parte activa, a niveles en los cuales el agua se vaporice y pueda ser extraída en forma de gas [5]. Al convertirse el agua en vapor [6], este puede ser evacuado rápidamente por medio de la bomba de vacío.

\section{METODOLOGÍA DE LA INVESTIGACIÓN}

La unidad de análisis de la investigación fue el grado de secado del aislamiento sólido de los arrollamientos del transformador de distribución. El tamaño de la muestra consistió de cuatro partes activas de transformadores trifásicos de distribución, de fabricación nacional, cuyas características técnicas generales se presentan en la Tabla 1.

Tabla 1. Características técnicas de los transformadores

\begin{tabular}{|c|c|c|c|c|c|c|}
\hline \multirow[b]{2}{*}{$\mathrm{N}^{\circ}$} & \multirow{2}{*}{$\begin{array}{c}\text { Potencia } \\
\text { Nominal } \\
\text { (KVA) }\end{array}$} & \multicolumn{2}{|c|}{ Tensión Nominal } & \multicolumn{2}{|c|}{$\begin{array}{c}\text { Tensión Máxima de } \\
\text { Servicio }\end{array}$} & \multirow{2}{*}{$\begin{array}{c}\text { Peso } \\
\text { Parte } \\
\text { Activa } \\
(\mathrm{Kg})\end{array}$} \\
\hline & & $\begin{array}{c}\text { Alta } \\
\text { Tensión } \\
(\mathrm{kV})\end{array}$ & $\begin{array}{c}\text { Baja } \\
\text { Tensión } \\
(\mathrm{kV})\end{array}$ & $\begin{array}{c}\text { Alta } \\
\text { Tensión } \\
(\mathrm{kV})\end{array}$ & $\begin{array}{c}\text { Baja } \\
\text { Tensión } \\
(\mathrm{kV})\end{array}$ & \\
\hline 1 & 500 & 22,90 & 0,46 & 36 & 3,6 & 783 \\
\hline 2 & 500 & 22,90 & 0,40 & 36 & 3,6 & 777 \\
\hline 3 & 500 & 22,90 & 0,46 & 36 & 3,6 & 714 \\
\hline 4 & 500 & 22,90 & 0,40 & 36 & 3,6 & 760 \\
\hline
\end{tabular}
Kilovoltioamperios. kV: Kilovoltios. Kg: Kilogramos.

Con la finalidad de determinar y analizar los factores que influyeron significativamente en el valor del factor de potencia del aislamiento de los arrollamientos, durante el tratamiento en vacío, se consideraron dos factores de influencia. El primer factor, fue el Aislamiento seco de los arrollamientos que comprendió tres niveles: a) Aislamiento seco del arrollamiento de baja tensión (Nivel de tensión máxima de servicio de 3,6 kV), b) Aislamiento seco entre arrollamientos de alta y baja tensión, c) Aislamiento seco del arrollamiento de alta tensión (Nivel de tensión máxima de servicio de $36 \mathrm{kV}$ ). El segundo factor, fue el Tiempo del tratamiento en vacío que comprendió cinco niveles, que fueron los intervalos de medición de media hora. El Factor de potencia del aislamiento, expresado en $\%$, fue la respuesta observada. De las pruebas se obtuvieron los siguientes datos: a) Las temperaturas del núcleo magnético de la parte activa de uno de los transformadores, medidos durante el secado en el horno; b) Los valores de la capacitancia de los arrollamientos de cada uno de los cuatro transformadores, medidos durante el tratamiento en vacío; c) Los valores del factor de potencia del aislamiento de los arrollamientos de cada uno de los

Revista TECNIA Vol. 28 N $^{\circ} 1$ Agosto - Diciembre 2018 
cuatro transformadores, medidos durante el tratamiento en vacío.

En el análisis de los resultados se aplicó el diseño factorial utilizando un software de análisis estadístico [7], que permitió contrastar las hipótesis de investigación y determinar la interacción entre factores y el efecto de los factores en la respuesta. Para ello se aplicó un análisis de varianza (ANOVA) de dos factores [8], el que contrasta, para cada uno de los factores, la hipótesis nula de que los resultados de la variable dependiente no dependen del factor.

\section{EXPERIMENTACIÓN}

Las cuatro partes activas de los transformadores seleccionados como muestras, fueron sometidas a un proceso de tratamiento térmico de secado en el horno - durante 72 horas y a presión atmosférica - con la finalidad de extraer la humedad de los aislamientos; habiéndose monitoreado la temperatura del núcleo magnético de uno de ellos, inicialmente en periodos de una hora, obteniéndose la curva de calentamiento que se presenta en la Figura 5. Se realizó el montaje de cada parte activa en su respectivo tanque (transformador completo), y luego de ello, el transformador se colocó dentro de un tanque diseñado para soportar presiones en vacío sellándose herméticamente contra el exterior.

Se inició al tratamiento en vacío con una presión de un milibar, manteniéndose constante durante todo el proceso que tuvo una duración de dos horas. En intervalos de media hora se midió el valor de las capacitancias de los arrollamientos y el factor de potencia del aislamiento, utilizando un instrumento para prueba de baja tensión en sistemas de aislamientos.
De la medición de la Capacitancia de los Arrollamientos de la parte activa de cada transformador se obtuvieron los resultados que se presentan en la Tabla 2. En ella se puede apreciar que los valores de las capacitancias se mantienen aproximadamente constantes, tanto para el aislamiento de los arrollamientos de alta tensión y de baja tensión como para el aislamiento entre ambos arrollamientos. Esto en razón de que el tratamiento en vacío no afecta significativamente la geometría y dimensiones de los arrollamientos de alta tensión y baja tensión de la parte activa del transformador de distribución.

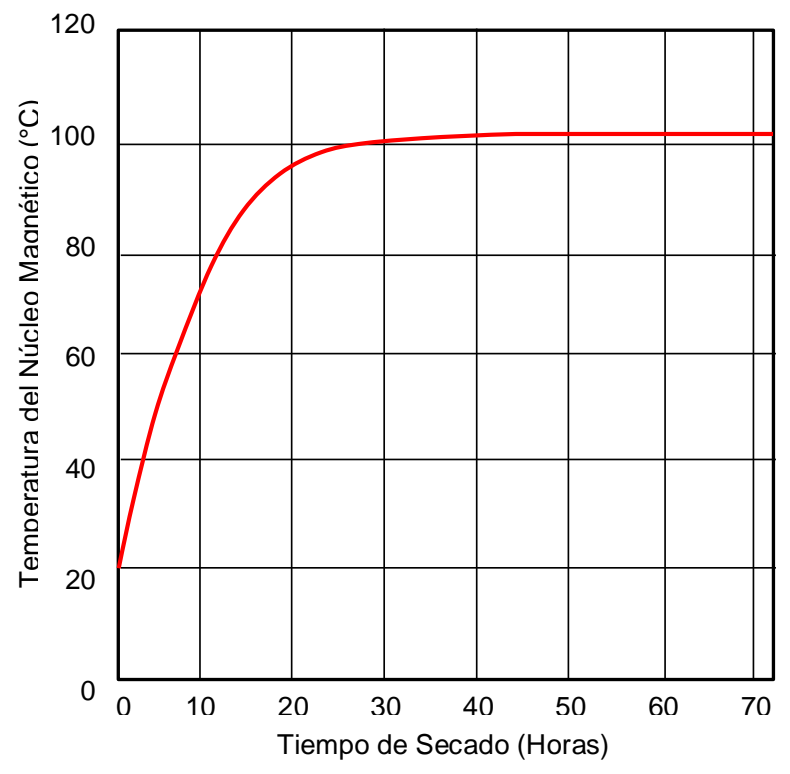

Figura 5. Curva de calentamiento del núcleo magnético de la parte activa del transformador de distribución.

Tabla 2. Valores medidos de la capacitancia de los arrollamientos

\begin{tabular}{|c|c|c|c|c|c|c|c|c|c|c|}
\hline \multirow{3}{*}{$\mathrm{N}^{\circ}$} & \multicolumn{4}{|c|}{ Prueba General } & \multicolumn{6}{|c|}{$\begin{array}{l}\text { Capacitancia de los Arrollamientos } \\
\text { (Picofaradios) }\end{array}$} \\
\hline & \multirow[t]{2}{*}{ Modo } & \multirow[t]{2}{*}{ Energizado } & \multirow[t]{2}{*}{ Guarda } & \multirow[t]{2}{*}{ UST } & \multirow{2}{*}{$\begin{array}{l}\text { Aislamiento del } \\
\text { Arrollamiento }\end{array}$} & \multicolumn{5}{|c|}{$\begin{array}{c}\text { Tiempo de Medición } \\
\text { (Horas del Tratamiento en Vacío) }\end{array}$} \\
\hline & & & & & & 0,0 & 0,5 & 1,0 & 1,5 & 2,0 \\
\hline \multirow{3}{*}{1} & GST & Alta Tensión & Baja Tensión & & $C_{H}:$ Alta Tensión & 388,25 & 388,40 & 387,80 & 387,60 & 387,40 \\
\hline & UST & Alta Tensión & & Baja Tensión & $\begin{array}{c}C_{H L}: \text { Alta y Baja } \\
\text { Tensión }\end{array}$ & 1161,80 & 1161,20 & 1160,40 & 1159,60 & 1159,00 \\
\hline & GST & Baja Tensión & Alta Tensión & & $C_{L}:$ Baja Tensión & 1322,20 & 1322,00 & 1321,60 & 1321,20 & 1320,80 \\
\hline \multirow{3}{*}{2} & GST & Alta Tensión & Baja Tensión & & $C_{H}$ : Alta Tensión & 393,20 & 391,45 & 391,45 & 391,80 & 394,55 \\
\hline & UST & Alta Tensión & & Baja Tensión & $\begin{array}{c}C_{H L}: \text { Alta y Baja } \\
\text { Tensión }\end{array}$ & 914,80 & 907,60 & 908,60 & 910,80 & 910,00 \\
\hline & GST & Baja Tensión & Alta Tensión & & $C_{L}:$ Baja Tensión & 1353,00 & 1152,00 & 1152,80 & 1154,00 & 1153,40 \\
\hline \multirow{3}{*}{3} & GST & Alta Tensión & Baja Tensión & & $C_{H}$ : Alta Tensión & 340,90 & 332,70 & 332,50 & 332,30 & 332,20 \\
\hline & UST & Alta Tensión & & Baja Tensión & $\begin{array}{c}C_{H L}: \text { Alta y Baja } \\
\text { Tensión }\end{array}$ & 819,60 & 814,60 & 813,80 & 813,20 & 812,60 \\
\hline & GST & Baja Tensión & Alta Tensión & & $C_{L}:$ Baja Tensión & 1362,00 & 1345,00 & 1344,40 & 1343,20 & 1342,20 \\
\hline 4 & GST & Alta Tensión & Baja Tensión & & $C_{H}:$ Alta Tensión & 427,40 & 419,50 & 416,70 & 415,70 & 415,00 \\
\hline
\end{tabular}




\begin{tabular}{|c|c|c|c|c|c|c|c|c|c|c|}
\hline & UST & Alta Tensión & & Baja Tensión & $\begin{array}{c}C_{H L} \text { : Alta y Baja } \\
\text { Tensión }\end{array}$ & 1262,00 & 1262,00 & 1261,00 & 1260,00 & 1259,40 \\
\cline { 2 - 9 } & GST & Baja Tensión & Alta Tensión & & $C_{L}$ : Baja Tensión & 1431,40 & 1428,40 & 1424,20 & 1422,40 & 1421,60 \\
\hline
\end{tabular}

$\mathrm{N}^{\circ}$ : Número del transformador de distribución sometido a ensayo.

De la medición del Factor de Potencia del Aislamiento de cada transformador, se obtuvieron los resultados que muestran en la Tabla 3; en la que se puede apreciar que los valores del factor de potencia (\%) correspondientes al aislamiento seco de los arrollamientos de alta tensión y de baja tensión, al inicio del tratamiento en vacío, son superiores a los obtenidos al final del tratamiento en vacío. Lo que demuestra la efectividad del proceso para eliminar la humedad remanente en los aislamientos de los arrollamientos de la parte activa del transformador de distribución. Así mismo se observa que el factor de potencia del aislamiento entre arrollamientos de alta y baja tensión no tiene una variación significativa durante el proceso.

Tabla 3. Valores medidos del factor de potencia del aislamiento

\begin{tabular}{|c|c|c|c|c|c|c|c|c|c|c|}
\hline \multirow{3}{*}{$\mathrm{N}^{\circ}$} & \multicolumn{4}{|c|}{ Prueba General } & \multicolumn{6}{|c|}{$\begin{array}{l}\text { Factor de Potencia del Aislamiento } \\
\qquad(\%)\end{array}$} \\
\hline & \multirow[t]{2}{*}{ Modo } & \multirow[t]{2}{*}{ Energizado } & \multirow[t]{2}{*}{ Guarda } & \multirow[t]{2}{*}{ UST } & \multirow{2}{*}{$\begin{array}{l}\text { Aislamiento del } \\
\text { Arrollamiento }\end{array}$} & \multicolumn{5}{|c|}{$\begin{array}{c}\text { Tiempo de Medición } \\
\text { (Horas del Tratamiento en Vacío) }\end{array}$} \\
\hline & & & & & & 0,0 & 0,5 & 1.0 & 1,5 & 2,0 \\
\hline \multirow{3}{*}{1} & GST & Alta Tensión & Baja Tensión & & Alta Tensión & 1,392 & 0,588 & 0,584 & 0,582 & 0,580 \\
\hline & UST & Alta Tensión & & Baja Tensión & Alta y Baja Tensión & 0,232 & 0,221 & 0,217 & 0,215 & 0,213 \\
\hline & GST & Baja Tensión & Alta Tensión & & Baja Tensión & 1,021 & 0,937 & 0,933 & 0,925 & 0,915 \\
\hline \multirow{3}{*}{2} & GST & Alta Tensión & Baja Tensión & & Alta Tensión & 1,180 & 0,839 & 0,829 & 0,826 & 0,825 \\
\hline & UST & Alta Tensión & & Baja Tensión & Alta y Baja Tensión & 0,263 & 0,196 & 0,190 & 0,179 & 0,175 \\
\hline & GST & Baja Tensión & Alta Tensión & & Baja Tensión & 1,050 & 0,985 & 0,976 & 0,970 & 0,950 \\
\hline \multirow{3}{*}{3} & GST & Alta Tensión & Baja Tensión & & Alta Tensión & 2,080 & 0,495 & 0,465 & 0,456 & 0,450 \\
\hline & UST & Alta Tensión & & Baja Tensión & Alta y Baja Tensión & 0,120 & 0,100 & 0,082 & 0,080 & 0,079 \\
\hline & GST & Baja Tensión & Alta Tensión & & Baja Tensión & 1,030 & 0,526 & 0,513 & 0,510 & 0,508 \\
\hline \multirow{3}{*}{4} & GST & Alta Tensión & Baja Tensión & & Alta Tensión & 1,840 & 0,720 & 0,603 & 0,541 & 0,507 \\
\hline & UST & Alta Tensión & & Baja Tensión & Alta y Baja Tensión & 0,123 & 0,123 & 0,118 & 0,117 & 0,116 \\
\hline & GST & Baja Tensión & Alta Tensión & & Baja Tensión & 0,638 & 0,556 & 0,535 & 0,528 & 0,524 \\
\hline
\end{tabular}

$\mathrm{N}^{\circ}$ : Número del transformador de distribución sometido a ensayo.

\section{ANÁLISIS E INTERPRETACIÓN DE RESULTADOS}

El paso previo a la contrastación de las hipótesis de investigación es verificar la validez del diseño factorial utilizado, mediante la prueba de significancia del análisis de varianza [8][7], cuyos resultados se presentan en la Tabla 4.

Tabla 4. Resultados del análisis de varianza

\begin{tabular}{|l|c|c|}
\hline \multicolumn{1}{|c|}{ Fuente de Variación } & $\begin{array}{c}\text { Estadístico } \\
\text { de Prueba } \\
F_{o}\end{array}$ & $\begin{array}{c}\text { Nivel de } \\
\text { Significancia } \\
p\end{array}$ \\
\hline $\begin{array}{l}\text { Factor Aislamiento Secos de los } \\
\text { Arrollamientos }\end{array}$ & 72,43 & $0,000^{*}$ \\
\hline $\begin{array}{l}\text { Factor Tiempo del Tratamiento } \\
\text { en Vacío }\end{array}$ & 10,83 & $0,000^{*}$ \\
\hline $\begin{array}{l}\text { Interacción entre Factores } \\
\text { Aislamiento Seco de los }\end{array}$ & 5,74 & $0,000^{*}$ \\
\hline
\end{tabular}

doi: https://doi.org/10.21754/tecnia.v28i2.349

\begin{tabular}{|l|l|l|}
\hline $\begin{array}{l}\text { Arrollamientos y Tiempo del } \\
\text { Tratamiento en Vacío }\end{array}$ & & \\
\hline$R^{2}=83,87 \% \quad R^{2}$ (corregido) $=78,86 \%$ & $\left(^{*}\right)$ & $p<0,05$ \\
\hline
\end{tabular}

De este análisis se puede apreciar que el valor de $\boldsymbol{R}^{2}$ (denominado coeficiente de determinación), indica que estadísticamente el $83,87 \%$ de la variabilidad de las mediciones del factor de potencia del aislamiento está explicada y sustentada, tanto por el efecto de los factores Aislamiento sólido de los arrollamientos y Tiempo del tratamiento en vacío, como por la Interacción entre ambos factores. Por tanto el diseño factorial es adecuado para el experimento.

Para la contrastación de las hipótesis con el análisis de varianza, se tiene que evaluar el nivel de significancia $\boldsymbol{p}$, que demuestra estadísticamente que se acepta la hipótesis de investigación si se cumple que su valor es $\boldsymbol{p}<\mathbf{0 , 0 5}$. Así, de los resultados de la contrastación de Revista TECNIA Vol. 28 NN$^{\circ} 1$ Agosto - Diciembre 2018 
las tres hipótesis, que se presentan en la Tabla 4, se infiere lo siguiente:

Se acepta la Hipótesis General $\boldsymbol{H}_{\mathbf{1}}$ y se puede afirmar que, para la muestra de transformadores de distribución, el aislamiento seco de los arrollamientos de la parte activa y el tiempo de tratamiento en vacío interactúan sobre el factor de potencia del aislamiento de los transformadores de distribución (Estadístico 5,74; significancia de 0,000; $p<0,05)$.

Se acepta la Hipótesis Específica $\boldsymbol{H}_{2}$ y se puede afirmar que, para la muestra de transformadores de distribución, el aislamiento seco de los arrollamientos produce efecto significativo sobre el factor de potencia del aislamiento de los transformadores de distribución (Estadístico 72,43; significancia de 0,000; $p<0,05$ ).

Se acepta la Hipótesis Específica $\boldsymbol{H}_{3}$ y se puede afirmar que, para la muestra de transformadores de distribución, el tiempo del tratamiento en vacío produce efecto significativo sobre el factor de potencia del aislamiento de los transformadores de distribución (Estadístico 10,83; significancia de 0,000; $p<0,05$ ).

La aceptación de las hipótesis se traduce en la interpretación práctica de los resultados, que se expone a continuación.

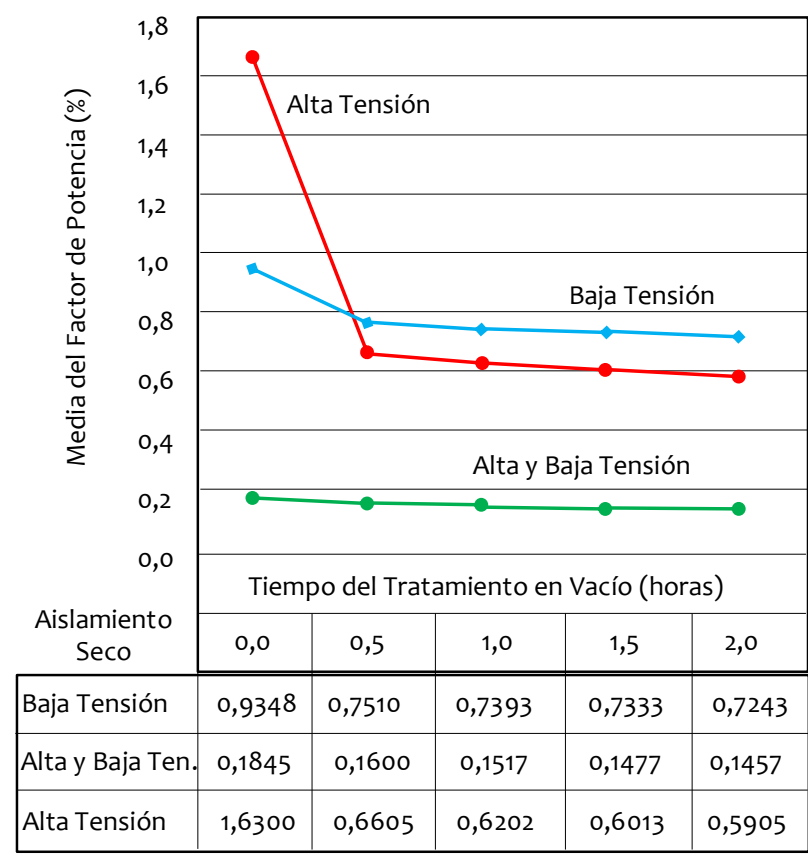

Figura 6. Gráfica de interacción entre factores. Medias ajustadas.

En la gráfica de interacción entre factores, de la Figura 6 , se observa que al cruzarse las líneas de los niveles del aislamiento seco - arrollamiento de alta tensión y arrollamiento de baja tensión aproximadamente a las 0,5 horas, se comprueba que existe interacción entre los factores Tiempo del

doi: https://doi.org/10.21754/tecnia.v28i2.349 tratamiento en vacío y Aislamiento seco de los arrollamientos; por tanto, la relación que existe entre la respuesta factor de potencia del aislamiento y el tiempo del tratamiento en vacío depende de la calidad del aislamiento seco de los arrollamientos.

En esta gráfica también se aprecia que, al iniciarse el tratamiento en vacío (0,0 horas), el aislamiento seco del arrollamiento de baja tensión tiene un valor promedio del factor de potencia menor al del arrollamiento de alta tensión; lo que significa que el aislamiento del arrollamiento de baja tensión por encontrarse cerca del núcleo magnético, que es la parte más caliente, ver Figura 7, ha tenido un secado uniforme en su aislamiento en comparación con el aislamiento del arrollamiento de alta tensión. Ello también podría indicar que el arrollamiento de alta tensión, al estar ubicado exteriormente con respecto al núcleo magnético, la exposición al medio ambiente, durante el proceso de ajuste y montaje de la parte activa en su tanque, ha originado que absorba humedad afectando la calidad del secado. En la misma gráfica, luego de 0,5 horas de tratamiento en vacío, el aislamiento del arrollamiento de alta tensión alcanza un valor promedio de factor de potencia que es menor al valor que tiene el arrollamiento de baja tensión; para luego mantenerse con una tendencia decreciente hasta el final del tratamiento en vacío.

Con relación a la línea correspondiente al nivel del aislamiento seco entre arrollamientos de alta y baja tensión, se observa que esta no se cruza con las otras líneas durante todo el tratamiento en vacío - valor promedio de factor de potencia del aislamiento aproximadamente constante - lo que significa que para este tipo de aislamiento no existe interacción entre los factores Tiempo del tratamiento en vacío y Aislamiento seco de los arrollamientos; ya que el tratamiento en vacío no afecta en gran medida el secado de estos aislamientos. Esto se explica porque en la zona intermedia de los arrollamientos (entre alta y baja tensión) el volumen de masa del aislamiento sólido es mínimo, puestos que, básicamente, son canales de refrigeración constituidos por cilindros aislantes y distanciadores axiales, como se aprecia en la Figura 7, y por tanto la escasa humedad que hubieren contenido ha sido eliminada rápidamente durante el secado de la parte activa en el horno. Este espacio posteriormente será ocupado por el aceite dieléctrico.

De la Figura 6 y Tabla 3 se determina, que el aislamiento seco de los arrollamientos representa un efecto individual importante sobre el factor de potencia del aislamiento. Ya que para los tres niveles del aislamiento, el factor de potencia disminuye gradualmente como consecuencia de la eliminación de la humedad. Lo que ha sido corroborado con el nivel de significancia $(p<0,05)$ obtenido en el análisis de

Revista TECNIA Vol. 28 N 1 Agosto - Diciembre 2018 
varianza, Tabla 4, y por tanto es un efecto en el que se debe priorizar su atención. Esto implica que el control del secado de la parte activa podría centrarse en el arrollamiento de alta tensión, puesto que, al ser de un nivel de tensión máxima de servicio de 36 kV y contar con mayor número de espiras, tiene también mayor masa de aislamiento sólido, ver Figura 8, en comparación con el arrollamiento de baja tensión, ver Figura 9.

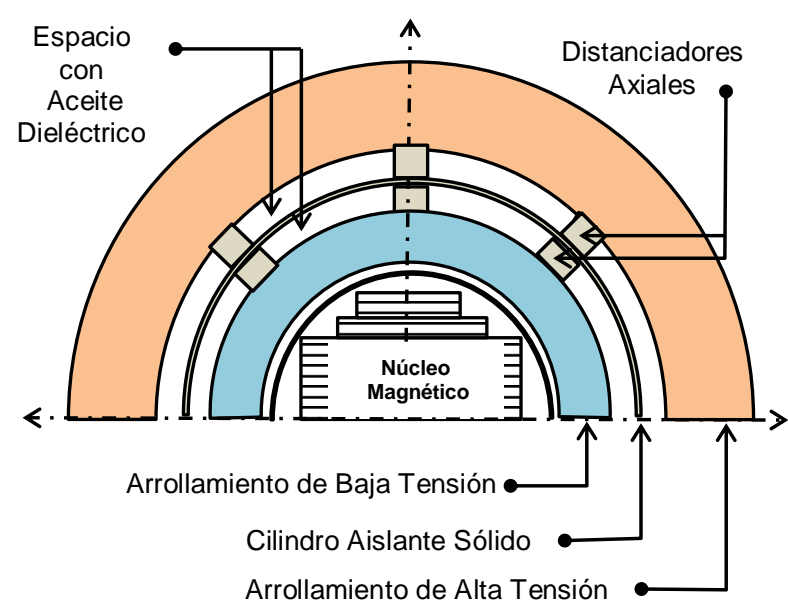

Figura 7. Vista transversal de los arrollamientos de la parte activa del transformador.

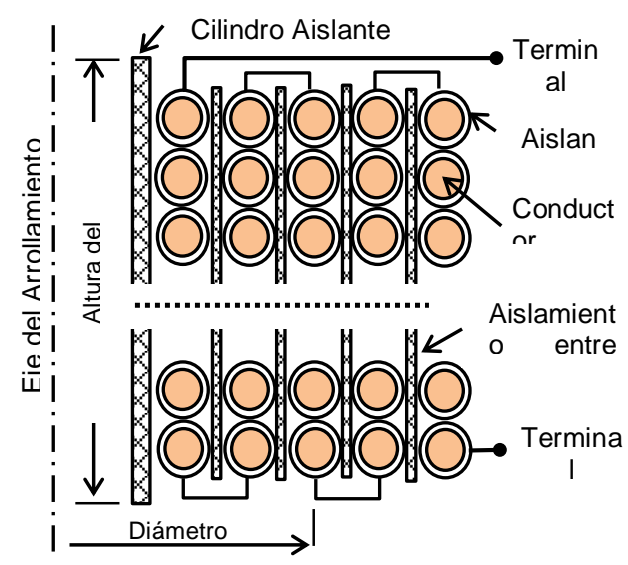

Figura 8. Disposición del aislamiento sólido en el arrollamiento de alta tensión (36 kV).

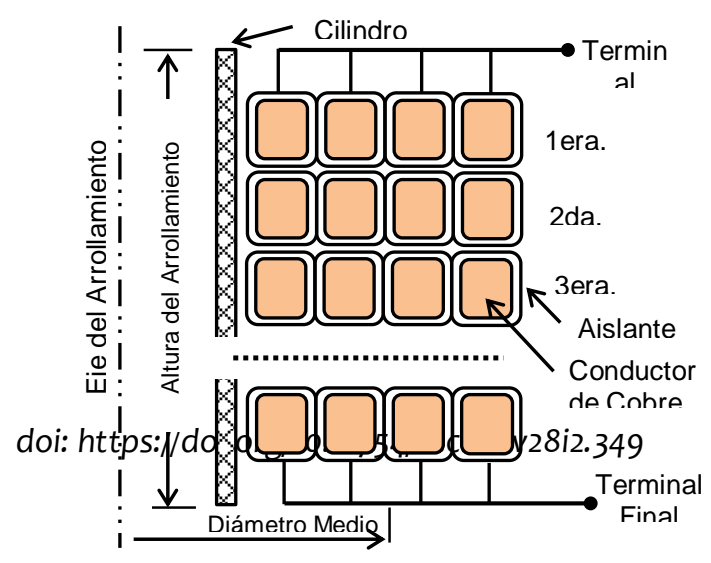

Figura 9. Disposición del aislamiento sólido en el arrollamiento de baja tensión $(3,6 \mathrm{kV})$.

De la Figura 6 y Tabla 3 también se determina, que el tiempo del tratamiento en vacío representa un efecto individual importante sobre el factor de potencia del aislamiento. Ya que para los cinco niveles de tiempo, el factor de potencia disminuye gradualmente conforme se acelera la evaporación del agua del aislamiento, Figura 10. Lo que ha sido corroborado con el nivel de significancia $(p<0,05)$ obtenido en el análisis de varianza, Tabla 4. Por tanto es recomendable que el tiempo del tratamiento en vacío sea como mínimo de dos horas, puesto que el efecto en el factor de potencia del aislamiento es favorable, alcanzándose un valor próximo al 0,5\%.

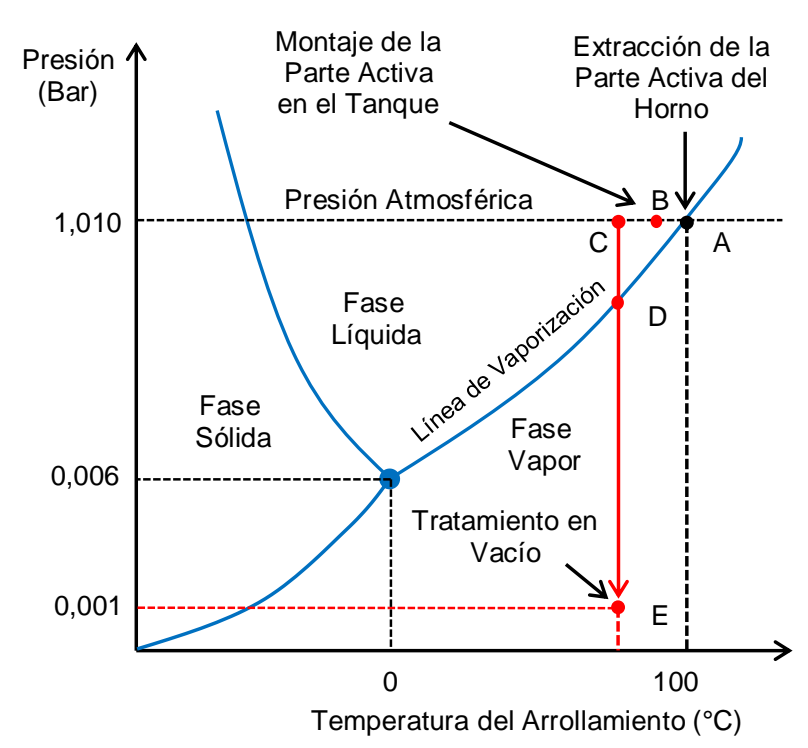

Figura 10. Diagrama de fases de la eliminación del agua contenida en el aislamiento. Adaptado de Fundamentos de Termodinámica [6].

Esto se explica porque al iniciarse el tratamiento en vacío, punto $\mathbf{C}$ de la Figura 10 , se produce una expansión del aire contenido dentro del tanque del transformador. Esta expansión ayuda a la expulsión inicial de la humedad, punto D. Simultáneamente, al reducirse el punto de ebullición del agua contenida en los aislamientos, la evaporación se acelera hasta que se logra la presión de un milibar, punto E. Así, conforme se

Revista TECNIA Vol. 28 Nº 1 Agosto - Diciembre 2018 
prolonga el tratamiento en vacío a presión constante, se mejora permanentemente el secado del aislamiento de los arrollamientos.

\section{CONCLUSIONES}

El principal aporte del estudio ha sido comprobar la influencia del tratamiento de secado en vacío sobre el factor de potencia del aislamiento de los arrollamientos de transformadores de distribución. Probándose estadísticamente, para la muestra en estudio, la hipótesis general y las hipótesis específicas formuladas en la investigación, demostrándose así la eficacia del proceso.

Se confirmó como efectos principales, sobre el factor de potencia del aislamiento de los arrollamientos, el grado de calidad del aislamiento seco de los arrollamientos y el tiempo de duración del tratamiento en vacío.

Se demostró, que el tiempo de duración del tratamiento en vacío produce un efecto que contribuye con la aceleración de la eliminación de la humedad remanente del aislamiento de los arrollamientos, traduciéndose en una disminución del valor promedio del factor de potencia del aislamiento.

Se demostró, que la calidad del secado del aislamiento sólido de los arrollamientos de alta tensión y baja tensión produce un efecto que se refleja en el valor promedio del factor de potencia del aislamiento.

Se comprobó, que la capacitancia a tierra de los arrollamientos de alta tensión y de baja tensión no se ve influenciada por el tratamiento en vacío.

\section{REFERENCIAS}

[1] Prueba de Tangente Delta y Capacidad Ingeniería de Máquinas Eléctricas (IME)

http://www.ime.com.co/filedownload/downloadfile/fileid/48/src/ @random558829acd336e

[2] Iwanusiw O W 2010 Insulation Power Factor Testing of Power Transformers Eltel Industries, Bangalore http://www.eltelindustries.com/wpcontent/uploads/2013/10/ACTS125K-PLUS.pdf

[3] IEEE Std 621995 IEEE Guide for Diagnostic Field Testing of Electric Power Apparatus- Part 1: Oil Filled Power Transformers, Regulators, and Reactors Revision of IEEE Std 62-1978

[4] IEEE Std C57.12.1999 IEEE Standard Test Code for Liquid-Inmersed Distribution, Power, and Regulating Transformers Revision of IEEE Std C57.12.90-1993

[5] Procedimiento para secado de transformadores de distribución Bombas de Vacío y Deshidratación S A http://www.bombasdevacio.com.mx/desgas_procedsecadotran sformadoresDistrib.html

[6] Van Wylen G y Sonntang R 1975 Fundamentos de Termodinámica (Primera Edición) México: Editorial Limusa pp 66-69

[7] Manual Minitab 16 Meet Minitab 16 https://es.scribd.com/doc/58634097/Manual-Minitab-16

[8] Montgomery D 2008 Diseño y Análisis de Experimentos (Segunda Edición) México: Limusa Wiley pp 170-200
Los artículos publicados por TECNIA pueden ser compartidos a través de la licencia Creative Commons: CC BY 4.0 Perú. Permisos lejos de este alcance pueden ser consultados a través del correorevistas@uni.edu.pe 